EPJ Web of Conferences 27, 00001 (2012)

DOI: $10.1051 /$ epjconf/20122700001

C) Owned by the authors, published by EDP Sciences, 2012

\title{
Systematic Quantification of Uncertainties for Evaluated Prompt Fission Neutron Spectra and Multiplicities
}

\author{
Patrick Talou ${ }^{1, a}$, Mike Rising ${ }^{1,2}$, Toshihiko Kawano ${ }^{1}$, and Anil Prinja ${ }^{2}$ \\ 1 Theoretical Division, Los Alamos National Laboratory, Los Alamos, NM, USA \\ 2 Department of Nuclear Engineering, University of New Mexico, USA
}

\begin{abstract}
Uncertainties associated with evaluated average prompt fission neutron spectra and multiplicities are obtained for a suite of actinides in the Los Alamos model formalism. Systematics for the model input parameters are taken from the literature and used as prior values in a Bayesian updating procedure. Posterior systematics as well as associated posterior uncertainties are inferred. In addition, cross-isotope correlations are evaluated for the first time. The quantification of uncertainties associated with advanced Monte Carlo Hauser-Feshbach calculations of prompt fission neutron spectra is also discussed.
\end{abstract}

\section{Introduction}

Recent years have seen a surge in the interest of quantifying uncertainties associated with evaluated nuclear data. Thanks to ever increasing computing power, running one or a hundred nuclear reaction simulations for data evaluation purposes is easily achievable by most evaluators. Studying the sensitivity of the results to (modest) changes in model parameter values is therefore possible and provides a relatively straightforward access to evaluated uncertainties (see for example large-scale TALYS runs [1]).

Obviously, performing large-scale nuclear reaction simulations does not provide fully adequate uncertainties associated with evaluated data. Since an uncertainty is not a physical quantity, but is instead an information on how well we know a physical datum, quantifying this uncertainty requires to assess all sources of uncertainties originating in the evaluation process itself. In an ideal situation, all the information that entered into the evaluation should be reflected in the associated uncertainty.

Nuclear data evaluations are often the result of a combination of partial experimental measurements and theoretical model calculations, which carry with them some uncertainties. In addition, it has been a common practice to adjust the results to match integral or semi-integral benchmark better. While this adjustment is usually performed within evaluated error bars, it is never accounted for in the uncertainty quantification (UQ) process. The added information is therefore included to modify the central values, without touching the associated uncertainties and correlations. We will discuss this issue again in the conclusion.

In the present paper, we discuss our recent work on evaluating prompt fission neutron spectrum (PFNS) and multiplicity (PFNM) for a suite of uranium isotopes, and on quantifying their uncertainties consistently. This is done in the framework of the Los Alamos model developed by Madland and Nix [2]. Prior model input parameters are obtained from established systematics, and a Bayesian inference scheme is used to combine model calculations and

\footnotetext{
a e-mail: talou@lanl.gov
}

known experimental data. By using systematics as a function of isotope and incident neutron energy, correlations appear between isotopes as well as between energies.

The Bayesian inference scheme is presented in Section 2 . Section 3 contains a brief introduction to the physics of prompt fission neutrons and gamma rays, as well as current theoretical models used to describe them. In particular, the Los Alamos (LA) model [2] used in the present work is discussed at some length. Recent work on advanced modeling of prompt fission neutrons and gamma rays is also briefly introduced, as it constitutes a natural extension of the present work. Some details on experimental efforts to measure PFNS and PFNM are discussed in Section 3.2. In the course of this work, a new evaluation code package was developed and is discussed briefly in Section 3.3. Numerical results for the suite of uranium isotopes studied here are presented in Section 4.3. Finally, we conclude by a discussion of the results and an outlook on the next steps to improve the current work for PFNS, and beyond.

\section{Quantifying PFNS Uncertainties}

Our approach to quantifying uncertainties on prompt fission neutron spectrum and multiplicity is very similar to the one we have used successfully for reaction cross sections [5]. It was described at some length in Ref. [6], but we are presenting it here briefly again for the sake of completeness.

A Bayesian approach is used to combine experimental and model parameter uncertainties. The equations for the Bayesian updating scheme used are as follows:

$$
\begin{aligned}
\mathbf{m}_{\mathbf{1}} & =\mathbf{m}_{\mathbf{0}}+\mathbf{P C}^{t} \mathbf{V}^{-1}\left(\phi-f\left(\mathbf{m}_{\mathbf{0}}\right)\right) \\
& =\mathbf{m}_{\mathbf{0}}+\mathbf{X} \mathbf{C}^{t}\left(\mathbf{C X C} \mathbf{C}^{t}+\mathbf{V}\right)^{-1}\left(\phi-f\left(\mathbf{m}_{\mathbf{0}}\right)\right), \\
\mathbf{P} & =\left(\mathbf{X}^{-1}+\mathbf{C}^{t} \mathbf{V}^{-1} \mathbf{C}\right)^{-1} \\
& =\mathbf{X}-\mathbf{X C}^{t}\left(\mathbf{C X C} \mathbf{C}^{t}+\mathbf{V}\right)^{-1} \mathbf{C X} .
\end{aligned}
$$

In those equations, $\mathbf{m}_{\mathbf{0}}$ represents the vector of prior model parameters, and $\mathbf{m}_{\mathbf{1}}$ is the vector of posterior model parameters. The quantity $f\left(\mathbf{m}_{\mathbf{0}}\right)$ is the calculated spectrum using 
prior parameter values. $\phi$ is the experimental spectrum. $\mathbf{X}$ and $\mathbf{P}$ are the prior and posterior model parameter covariance matrices, respectively. Finally, $\mathbf{V}$ represents the experimental covariance matrix, and $\mathbf{C}$ the sensitivity matrix whose elements are given by

$$
C_{i j}=\frac{\partial N\left(E_{i}\right)}{\partial p_{j}} \times \frac{p_{j}}{N\left(E_{i}\right)}
$$

at the energy $E_{i}$ for the parameter $p_{j}$. The evaluated covariance matrix $\mathbf{F}$ for the prompt neutron spectrum is finally obtained as

$$
\mathbf{F}=\mathbf{C P C}^{t}
$$

\section{Prompt Fission Neutrons}

In the present work, we refer to prompt fission neutrons and gamma rays as those emitted from the fission fragments formed right after the break up of a heavy nucleus. Those prompt particles are emitted within about $10^{-18}$ to $10^{-7} \mathrm{sec}$ after scission [7], until fission products are formed in their ground-state or long-lived isomeric states. Those products may eventually decay by weak interaction, and lead to the production of additional $\beta$-delayed neutrons and gamma rays.

Most modern evaluated nuclear data libraries contain information on the average prompt fission neutron spectrum (PFNS) and average prompt fission neutron multiplicity (PFNM) as a function of the incident neutron energy. Those quantities are commonly evaluated using the so-called Madland-Nix or Los Alamos (LA) model [2], or a simpler representation such as a Watt or Maxwellian spectrum, or a combination thereof.

More recently, Monte Carlo studies following the deexcitation of the primary fission fragments by successive emissions of neutrons and gamma rays have been developed successfully (for example, see Ref. [8]) and will be used in upcoming revisions of evaluated libraries.

\subsection{Theory}

\subsubsection{Los Alamos Model}

Prompt fission neutron spectra in the ENDF/B-VII.0 library were evaluated using the Los Alamos model [2]. Because the uncertainty quantification methodology follows the one for the evaluation of the PFNS in the first place, we recall here the major results of the Los Alamos model and identify the model parameters that enter in the following sensitivity calculations. We refer the interested reader to Ref. [2] for more details.

The neutron energy spectrum $N(E)$, in the laboratory frame, for a fission fragment moving with a kinetic energy per nucleon $E_{f}$ reads

$$
\begin{array}{r}
N(E)=\frac{1}{2 \sqrt{E_{f}} T_{m}^{2}} \int_{\left(\sqrt{E}-\sqrt{E_{f}}\right)^{2}}^{\left(\sqrt{E}+\sqrt{E_{f}}\right)^{2}} d \epsilon \sigma_{c}(\epsilon) \sqrt{\epsilon} \\
\times \int_{0}^{T_{m}} d T k(T) T \exp (-\epsilon / T),
\end{array}
$$

where $\epsilon$ is the center-of-mass neutron energy, $k(T)$ is the temperature-dependent normalization constant given by

$$
k(T)^{-1}=\int_{0}^{\infty} d \epsilon^{\prime} \sigma_{c}\left(\epsilon^{\prime}\right) \epsilon^{\prime} \exp \left(-\epsilon^{\prime} / T\right) .
$$

$\sigma_{c}\left(\epsilon^{\prime}\right)$ is the energy-dependent cross section for the inverse process of compound nucleus formation, and $T_{m}$ is the maximum value of the triangular distribution of temperatures used to describe the excitation energy distribution in the fragments. Considering the most probable fragmentation only, the average neutron energy spectrum in the laboratory frame is given by a simple average over the spectra for the light and heavy fragments. Following the original formulation of the LA model, an equal number of neutrons emitted from the light and heavy fragments is assumed.

At higher incident energies, these formulas can be generalized to take into account the onset of multi-chance fission, i.e. the probability that one or more neutrons are emitted before the residual compound nucleus fissions. However, in the present work, we are only concerned with lowenergy $(0.5 \mathrm{MeV})$ fission for which only the first-chance fission component is non-zero.

\subsubsection{Monte Carlo Hauser-Feshbach}

In the Monte Carlo Hauser-Feshbach (MCHF) approach, as described in $[3,4]$, the de-excitation of the primary fission fragments is followed exactly for each neutron and gamma-ray emission using the Hauser-Feshbach formalism and emission probabilities obtained from optical model calculations for the neutrons and gamma-ray strength functions for the gamma rays. Such simulations require to know the fission fragment yields as a function of mass and charge of the fission fragment. Also, the initial excitation energy, spin and parity distributions in each decaying fragment has to be known. Once those initial conditions are fixed, Hauser-Feshbach calculations are performed at each stage of the decay, and characteristics of the emitted particles are recorded and analyzed.

A fundamental advantage of MCHF calculations compared to the LA model is that various exclusive data can be assessed, as opposed to only two average quantities, i.e., average PFNS and PFNM. Modern experiments can explore this much richer phase-space and shed some light on the very interesting physics near the scission point.

MCHF calculations to study fission fragment decay implies to use a large number of data and model parameters for a large number of nuclei. Those parameters are the same as those that appear in standard Hauser-Feshbach calculations, and can be probed in various ways. One of them is the so-called Total Monte Carlo (TMC) approach as described in Ref. [1]. Besides those parameters, uncertainties in the determination of the primary fission fragment yields have also to be taken into account.

\subsection{Experiments}

Recently, significant experimental efforts have been initiated to improve our knowledge of prompt fission neutrons and in particular to obtain an accurate determination of 
their average spectrum [9-12]. These renewed efforts come with the realization that uncertainties in evaluated spectra can lead to significant uncertainties in integral quantities that are relevant to many nuclear applications.

In the present work, experimental covariance matrices were estimated in a very simplified fashion, and no effort has been made to get a more realistic representation of the systematic uncertainties. In particular, known crosscorrelations between measurements and isotopes have not been taken into account. This is a clear starting point for improving the preliminary results presented here.

\subsection{Evaluation}

In the course of this work, we have developed an evaluation package for analyzing and predicting PFNS and PFNM. Written in modern Fortran, it is made up of various modules that can be used to perform LA model calculations and parameter sensitivity studies, read experimental data and uncertainties, perform Bayesian statistics, create probability distribution functions of model parameters, generate covariance matrices, and read and write ENDF-formatted prompt fission neutron spectrum, multiplicity and covariance matrices. It also contains a module for producing highquality figures easily.

It represents a powerful tool to perform any serious study related to the evaluation of prompt fission neutrons. Plans to expand its use to MCHF calculations are in development.

\section{Study of a Suite of Uranium Isotopes}

In the following, numerical results are presented for a suite of uranium isotopes, for which the LA model was used.

\subsection{Model Parameter Systematics}

In order to study a suite of uranium isotopes, we have used the model parameter systematics of Tudora [13] as prior values in the Bayesian analysis. At low incident neutron energies, i.e., below the threshold for second-chance fission, the most important input parameters entering in LA model calculations are the average Total Kinetic Energy $\langle T K E\rangle$, the average energy release $\left\langle E_{r}\right\rangle$, the average level density parameter $\langle a\rangle$, and the average neutron separation energy $\left\langle S_{n}\right\rangle$. In theory, $\left\langle E_{r}\right\rangle$ can be obtained from calculating the $Q$-value of the fission reaction for each fission fragment pair appearing in the observed mass and charge yields, and $\left\langle S_{n}\right\rangle$ can also be derived approximately from mass difference calculations over the entire chain of prompt neutron decay. However, the large number of unknowns leaves room for an adjustment on those parameters, and can be considered free within known limits.

By studying a large set of isotopes, Tudora has inferred [13] fitted formulae for those parameters at thermal neutron energy. They are of the following form:

$$
\begin{aligned}
\langle T K E\rangle_{t h} & =\alpha_{1}+\alpha_{2} Z^{2} / A^{1 / 3}, \\
\left\langle E_{r}\right\rangle_{t h} & =\alpha_{3}+\alpha_{4} x+\alpha_{5} x^{2}, \\
\left\langle S_{n}\right\rangle_{t h} & =\alpha_{6}+\alpha_{7} x+\alpha_{8} x^{2}, \\
\langle a\rangle & =A / \alpha_{9},
\end{aligned}
$$

where $x$ is the fissility parameter given by

$$
x=\frac{Z^{2}}{A}\left[50.883\left(1-1.7826 \frac{(N-Z)^{2}}{A^{2}}\right)\right] .
$$

Numerical values for the $\left\{\alpha_{i}\right\}$ parameters can be found in [13] for suites of isotopes, and will not be repeated here.

Tudora also includes a slight linear dependence of the average Total Kinetic Energy $\langle T K E\rangle$ as a function of the incident neutron energy. At the lowest incident energies, it results in a very small change only. In this work, we do not include any energy dependence of the LA model parameters.

By starting from model parameter systematics, we are shifting the problem from the suite of parameters $\left\{\left\langle T K E^{i}\right\rangle,\left\langle E_{r}^{i}\right\rangle,\left\langle S_{n}^{i}\right\rangle,\left\langle a^{i}\right\rangle\right\}_{i=1 . . N}$, where $N$ is the number of isotopes considered, to a set of nine parameters $\left\{\alpha_{1}, \cdots, \alpha_{9}\right\}$ that determine the LA model parameter values for all isotopes at once. It allows a consistent evaluation of PFNS for a suite of isotopes, even for those with no existing measurement, thereby building correlations along the chain.
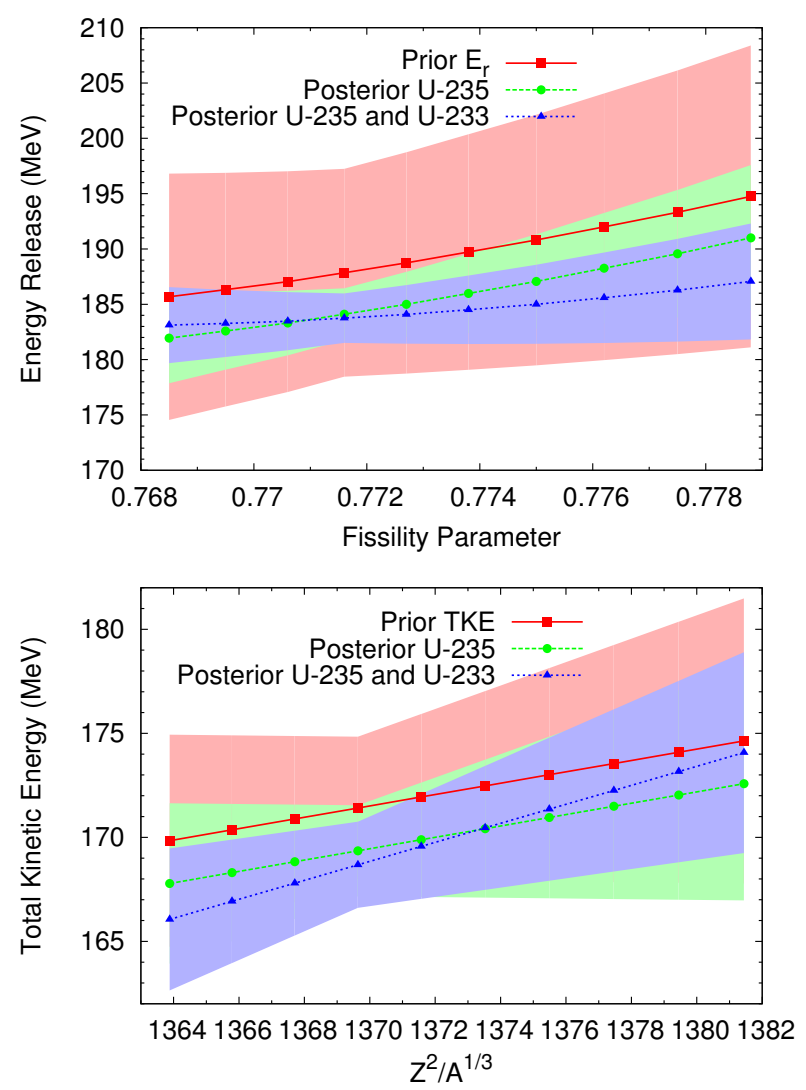

Fig. 1. Values of the model input parameters $\left\langle E_{r}\right\rangle$ and $\langle T K E\rangle$ appearing in the Los Alamos model. Prior values were taken from Tudora [13] and prior uncertainties were grossly estimated based on previous work [6]. Posterior values were obtained using ${ }^{235} \mathrm{U}$ experimental data first, then adding other data on ${ }^{233} \mathrm{U}$ thermal PFNS.

\subsection{Experimental Data}

Experimental data for the suite of uranium isotopes were collected and a selection of those is summarized in Table 1. 
Experimental results exist for a subset of uranium isotopes only, demonstrating the interest in studying all isotopes at once and bringing correlations across isotopes.

Table 1. Experimental database on prompt fission neutron spectra for the suite of uranium isotopes studied.

\begin{tabular}{cccccc}
\hline Isotope & EXFOR & 1st Author & Year & $E_{\text {inc }}$ & Ref. \\
\hline U-233 & $22688-002$ & Miura & 2002 & $0.55 \mathrm{MeV}$ & {$[14]$} \\
& $30704-002$ & Lajtai & 1985 & thermal & {$[15]$} \\
& $40872-005$ & Starostov & 1983 & thermal & {$[16]$} \\
\hline U-235 & - & Hambsch & 2010 & thermal & {$[10]$} \\
& $30704-003$ & Lajtai & 1985 & thermal & {$[15]$} \\
& $40872-004$ & Starostov & 1983 & thermal & {$[16]$} \\
& $40873-001$ & Bojcov & 1983 & thermal & {$[17]$} \\
& $20175-001$ & Johansson & 1977 & $0.53 \mathrm{MeV}$ & {$[18]$} \\
& $20996-003$ & Adams & 1975 & $0.52 \mathrm{MeV}$ & {$[19]$} \\
\hline
\end{tabular}

Note that several of these measurements are highly correlated since they were performed in the same experiment, e.g., Lajtai et al. measurements of ${ }^{233} \mathrm{U}$ and ${ }^{235} \mathrm{U}$ spectra. In the present work, no cross-isotope or cross-measurement correlation was considered. It is a clear deficiency of this work, but not one that cannot be handled in the future. The formalism and codes presented here can indeed very well be adjusted to take into account those cross-correlations appropriately.

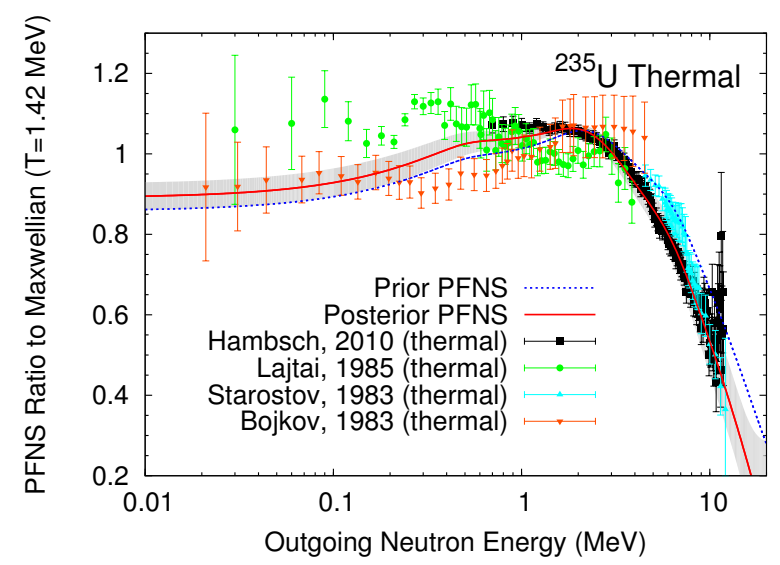

Fig. 2. Prior and posterior calculated prompt fission neutron spectra for the thermal neutron-induced fission reaction on ${ }^{235} \mathrm{U}$, and compared with experimental data.

\subsection{Numerical Results}

In [13], Tudora provides mean values for the main model input parameters, as described above. We used those values as prior estimates in our Bayesian inference tools. Prior uncertainties were grossly estimated, as they do not influence much the final answers. Fig. 1 shows those parameter values and uncertainty bands as a function of $Z^{2} / A^{1 / 3}$ or the fissility parameter $x$.

Posterior model parameter values and uncertainties were obtained by applying Eqs. (1) and (2) to the $\left\{\alpha_{i}\right\}$ model parameters. First, only experimental data sets on ${ }^{235} \mathrm{U}$ were used. Those already helped reduce the uncertainties for some parameters. Parameters related to the slopes of the mass-dependent formulae in Eq. (7) were not impacted by the inclusion of ${ }^{235} \mathrm{U}$ data only. A second result was obtained by including ${ }^{233} \mathrm{U}$ data in addition to ${ }^{235} \mathrm{U}$ data. This time, all parameters were modified. Note that the final standard deviations were scaled-up, as the raw KALMAN results led to very small uncertainties. This is a known problem that impacts all least-square results when correlations are missing or underestimated. A preliminary scaling factor of 5.0 was used in the figures below, but a more accurate result would be obtained using the $\chi^{2} / N$ between experimental and calculated spectrum values.

Fig. 2 shows results obtained for the ${ }^{235} \mathrm{U}$ thermal PFNS. The prior spectrum obtained with prior parameters inferred from Tudora systematics is shown to be very hard and deviates significantly from the experimental data sets. The posterior spectrum follows closely the recent measurement by Hambsch [10]. This comes as no surprise as those data have the smallest quoted uncertainties, and thus the biggest weight in our statistical analysis.

The thermal neutron-induced PFNS for ${ }^{233} \mathrm{U}$ was also modified in this global evaluation, and the result is shown in Fig. 3. In this case the posterior PFNS is mainly due to experimental data for ${ }^{235} \mathrm{U}$.

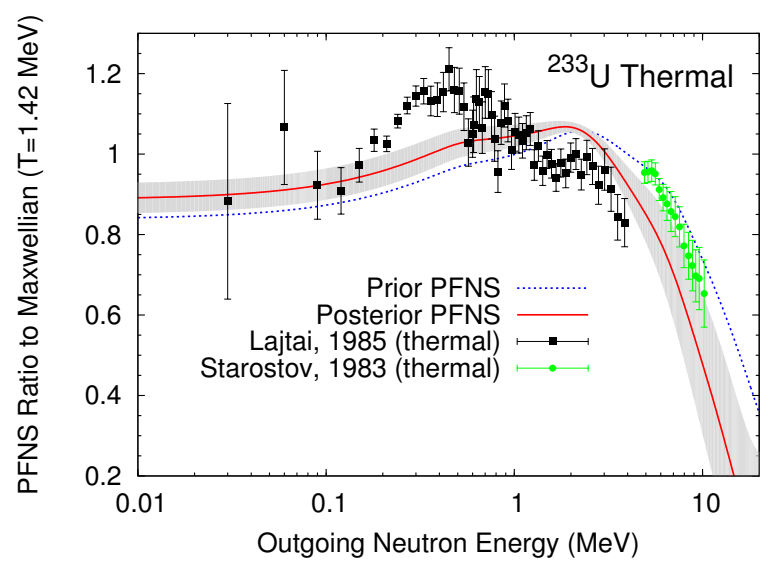

Fig. 3. Prior and posterior calculated prompt fission neutron spectra for the thermal neutron-induced fission reaction on ${ }^{233} \mathrm{U}$, and compared with experimental data. Note that experimental data sets are normalized to the prior PFNS, not the posterior.

In fact all evaluated PFNS for the uranium suite were updated through the use of the posterior model parameter values $\left\{\alpha_{1}, \cdots, \alpha_{9}\right\}_{\text {post }}$. While prior values and uncertainties for those parameters were assumed to be uncorrelated, the final correlation matrix shows significant non-diagonal factors:

\begin{tabular}{|c|c|c|c|c|c|}
\hline \multicolumn{6}{|l|}{100} \\
\hline \multicolumn{6}{|c|}{2100} \\
\hline 44 & $\begin{array}{ll}-3 & 100\end{array}$ & & & & \\
\hline-10 & $78-2$ & 100 & & & \\
\hline 0 & 2 & -2 & 00 & & \\
\hline 0 & 0 & 0 & & & \\
\hline 0 & 0 & 0 & 0 & & \\
\hline 0 & 0 & 0 & 0 & 0 & 0100 \\
\hline 39 & $6-65$ & -8 & 0 & 0 & $\begin{array}{lll}0 & 0 & 100\end{array}$ \\
\hline
\end{tabular}


In particular, as expected the coefficients $\alpha_{1}$ and $\alpha_{3}$, as well as $\alpha_{4}$, are strongly correlated to keep the effective temperature in the Los Alamos model equations within a reasonable range of values. A strong anti-correlation is also noted between $\alpha_{3}$ and $\alpha_{9}$, i.e., between the average energy release and the average level density parameter. Those correlations had already been noted in [6].

\section{Conclusion and Outlook}

The results presented here represent a natural extension of our previous work on the quantification of uncertainties for the evaluated PFNS on $\mathrm{n}(0.5 \mathrm{MeV})+{ }^{239} \mathrm{Pu}$ [6]. By studying more than one isotope and one incident neutron energy at a time, we are able to update evaluated PFNS even for those isotopes that have no associated measured data. It provides a more natural and encompassing framework not only for the quantification of uncertainties but also for the evaluations of prompt fission neutron spectra in general.

Several limitations of this preliminary work should be emphasized. First and foremost, the experimental covariance matrices used in the present study are rudimentary in their derivation. In addition no cross-correlations between data sets were considered, where they obviously exist. For instance, two Lajtai measurements [15] for ${ }^{233} \mathrm{U}$ and ${ }^{235} \mathrm{U}$ were performed in the very same experiment, and significant correlations in the determination of the experimental PFNS are expected.

Another limitation is the use of low-energy spectrum data only, while more data exist at higher incident neutron energies and even more data on the average neutron multiplicity $\bar{v}\left(E_{\text {inc }}\right)$. Such data could easily be included in a further analysis using the exact same tools.

Finally, the Los Alamos model to represent a prompt fission neutron spectrum is based on several hypotheses and approximations that could now be lifted. In particular, Monte Carlo Hauser-Feshbach (MCHF) techniques are being developed $[3,4,8]$ to study the de-excitation stage of the primary fission fragments in a much more detailed way. Such techniques can compute not only the average spectrum and multiplicity, as are obtained in the LA model, but also a wide array of physical quantities inaccessible with a simpler approach: $\mathrm{P}(v), \bar{v}(\mathrm{~A}, \mathrm{Z}, \mathrm{TKE})$, exclusive spectra, $\mathrm{n}$ $\mathrm{n}$ energy and angular correlations, etc. Experimental data exist for some of them, which could further constrain these more sophisticated calculations. Monte Carlo techniques such as described in [1] could be used to probe the large model parameter phase space, and infer realistic spectrum uncertainties.

\section{References}

1. D. Rochman, A.J. Koning, S.C. van der Marck, A. Hogenbirk and D. van Veen, Journal of the Korean Physical Society 59 No. 2, (2011) 1236

2. D.G. Madland and J.R. Nix, Nucl. Sci. Eng. 81, (1982) 213

3. T. Kawano, P. Talou, and M.B. Chadwick, EPJ Web of Conferences 21, (2012) 04001

4. P. Talou, B. Becker, T. Kawano, and Y. Danon, EPJ Web of Conferences 21, (2012) 08003
5. P. Talou, P.G. Young, T. Kawano, M. Rising, and M.B. Chadwick, Nuclear Data Sheets 112 No. 12, (2011) 3054

6. P. Talou, T. Kawano, D.G. Madland, A.C. Kahler, D.K. Parsons, M.C. White, R.C. Little, and M.B. Chadwick, Nucl. Sci. Eng. 166, (2010) 254

7. D.G. Madland, Nucl. Phys. A772, (2006) 113

8. P. Talou, B. Becker, T. Kawano, M.B. Chadwick, and Y. Danon, Phys. Rev. C83, (2011) 064612

9. S. Noda, R.C. Haight, R.O. Nelson, M. Devlin, J.M. O'Donnell, A. Chatillon, T. Granier, G. Belier, J. Taieb, T. Kawano, and P. Talou, Phys. Rev. C83, (2011) 034604

10. F.-J. Hambsch, private communication (2010)

11. A. Chatillon, T. Granier, J. Taieb, G. Belier, B. Laurent, S. Noda, R.C. Haight, M. Devlin, R.O. Nelson, and J.M. O'Donnell, Proc. of Seminar on Fission (World Scientific Publishing Co., 2010) 173

12. A.S. Vorobyev, O.A. Shcherbakov, Yu.S. Pleva, A.M. Gagarski, G.V Val'ski, G.A. Petrov, V.I. Petrova, and T.A. Zavarukhina, Nucl. Inst. Meth. A598, (2009) 795

13. A. Tudora, Annals of Nuclear Energy 36, (2009) 72

14. T. Miura, M. Baba, T. Win, M. Ibaraki, Y. Hirasawa, T. Hiroishi, and T.Aoki, J. Nucl. Sci. Tech., Suppl. 2 No. 1, (2002) 409

15. A. Lajtai, J. Kecskemeti, J. Safar, and P.P. Dyachenko, Proc. Int. Conf. on Nuclear Data for Basic and Applied Science, 13-17 May 1985, Santa Fe, USA (Eds. P.G. Young, R.E. Brown, G.F. Auchampaugh, P.W. Lisowski, and L. Stewart, Vol. 1, 1985) 613

16. B.I. Starostov, V.N. Nefedov and A.A. Boytzov, Proc. Conf. Neutron Physics, Kiev, Vol.2 (1984) 290

17. A.A. Bojcov, A.F. Semenov, and B.I.Starostov, Proc. Conf. Neutron Physics, Kiev, Vol.2 (1984) 294

18. P.I. Johansson, B. Holmqvist, and T. Wiedling, Nucl. Sci. Eng. 62, (1977) 695

19. J.M. Adams and P.I. Johansson, Proc. of the Conf. Nuclear Cross Sections and Technology, Washington, D.C., March 3-7, 1975 (Eds. R.A. Schrack and C.D. Bowman, Vol. 2, 1975) 631 